Pacific Journal of Mathematic 


\title{
HOMOMORPHISMS OF ANNIHILATOR BANACH ALGEBRAS
}

\author{
GREGORY F. BACHELIS
}

Let $A$ be a semi-simple annihilator Banach algebra, and let $\nu$ be a homomorphism of $A$ into a Banach algebra. In this paper we describe various continuity properties of $\nu$. Let (*) be the condition that $I \oplus \Re(I)=A$ for all closed two-sided ideals $I$, where $\Re(I)=\{x \mid I x=(0)\}$. If $\left(^{*}\right)$ holds, then we show that there exists a constant $K$ and a finite set of primitive ideals such that $\|\nu(x)\| \leqq K\|x\| \cdot\|y\|$ whenever $y x=x$ and $x$ is in the intersection of this finite set. If $\left(^{*}\right)$ does not hold, then essentially the same conclusion is true, but with the given norm replaced by one which is defined on a dense subset of $A$. If $A$ is a dual $B^{*}$-algebra, then $\nu$ is continuous on the socle.

We also consider the existence of unconditional decompositions in $A$. We show that (*) holds if and only if the minimal-closed two-sided ideals of $A$ form an unconditional decomposition for $A$.

Let $A$ and $B$ be Banach algebras, and let $\nu$ be a homomorphism of $A$ into $B$. In this paper we are concerned with deducing various continuity properties of $\nu$ when restrictions are placed only on $A$, and $B$ remains arbitrary. This problem has been considered by Bade and Curtis [2] for $A$ a commutative, regular semi-simple Banach algebra, by Cleveland [6] for $A$ a $B^{*}$-algebra, and by Johnson [9] for $A$ the algebra of bounded operators on a Banach space. In this paper we consider this problem for $A$ a semi-simple annihilator Banach algebra (see $\S 2$ for definitions), and we obtain results analogous to those of Bade and Curtis. Since annihilator algebras are in general noncommutative and only have identity when finitedimensional, techniques essentially different from those in [2] must be employed. The main technical device is a noncommutative version of the "Main Boundedness Theorem" of Bade and Curtis [6, Corollary 3.2].

The main theorems of this paper are found in $\S 5$. There we show (Theorem 5.1) that if $A$ is a semi-simple annihilator Banach algebra in which $I \oplus \Re(I)=A$ for all closed two-sided ideals $I$, e.g. if $A$ is a proper $H^{*}$-algebra [1], a dual $B^{*}$-algebra, or $l_{p}, 1 \leqq p<\infty$, and if $\nu$ is a homomorphism of $A$ into a Banach algebra, then there exists a constant $K$ and a finite set of primitive ideals such that $\|\nu(x)\| \leqq K\|x\| \cdot\|y\|$ whenever $y x=x$ and $x$ is in the intersection of this finite set. If $A$ is an arbitrary semi-simple annihilator Banach 
algebra, then we show that essentially the same conclusion holds if one replaces the original norm by a new one which is defined on a dense subset of $A$. In $\S 7$ we specialize to the case when $A$ is a dual $B^{*}$-algebra. We show that any homomorphism of $A$ into a Banach algebra is continuous on the socle and we obtain several decomposition theorems about the closure of the range of an isomorphism of $A$.

In $\S 3$ the question of the existence of unconditional decompositions in a semi-simple annihilator Banach algebra $A$ is considered. We show (Theorem 3.4) that the minimal-closed two-sided ideals form an unconditional decomposition for $A$ if and only if $I \oplus \Re(I)=A$ for all closed two-sided ideals $I$. We obtain a similar result for one-sided ideals when $A$ is assumed to have a proper involution. Both of these results follow from a technical theorem which is established about unconditional decompositions in Banach spaces. In $\S 4$ the structure of ideals and idempotents in $A$ is considered. We show that the socle is the set of elements possessing a left identity. In $\S 6$ we establish several properties of the separating ideal of a homomorphism of $A$ into a Banach algebra.

2. Preliminaries. For $A$ an algebra, $S \subset A$, define $\mathcal{Q}(S)=$ $\{x \mid x S=(0)\}$ and $\Re(S)=\{x \mid S x=(0)\}$. Let $I$ be a closed right ideal and let $J$ be a closed left ideal in a Banach algebra $A$. Then $A$ is an annihilator algebra if $\mathfrak{L}(I)=(0)$ only when $I=A$ and $\Re(J)=(0)$ only when $J=A$. The algebra is called dual if $\mathscr{R} \mathscr{L}(I)=I$ and $\mathscr{L} \mathscr{R}(J)=J$ for all such $I$ and $J$. Dual algebras were introduced by Kaplansky [10] and annihilator algebras by Bonsall and Goldie [4]. For the basic structure theorems concerning these algebras, the reader is referred to the texts of Rickart [14] or Naimark [12]. The definitions of terms not defined in this paper are those of [14].

If $A$ is a semi-simple annihilator Banach algebra, we will denote by II the set of primitive ideals, which Civin and Yood [5] have shown are the same as the maximal-closed two-sided ideals. We will denote the set of minimal-closed two-sided ideals by $\mathfrak{M}$, and the set of maximalmodular two-sided ideals by $\Xi$. The socle (the algebraic sum of the minimal left or right ideals) is denoted by $\mathrm{F}$. We will always assume that our Banach algebras are complex.

3. Unconditional decompositions. In this section we consider the question of when certain families of ideals of a semi-simple annihilator Banach algebra form an unconditional decomposition. Since much of this problem can be stated in a Banach space framework, we will work in that more general area whenever possible.

Throughout this section, we will always assume that $A$ is a 
Banach space and that $\mathscr{F}=\left\{I_{\alpha} \mid \alpha \in C\right\}$ is a collection of closed subspaces with dense linear span such that $I_{\alpha} \cap \mathrm{cl}\left(\sum_{\alpha \neq \beta} I_{\beta}\right)=(0)$ for all $\alpha \in C$, where $\sum I_{\beta}$ denotes the algebraic sum of $\left\{I_{\beta}\right\}$ and "cl" means "closure".

Definition 3.1. An element $x \in A$ is called $\mathscr{F}$-decomposable if $x=\sum_{\alpha} x_{\alpha}$, where $x_{\alpha} \in I_{\alpha}$ and convergence is with respect to the net of finite partial sums. We denote the set of $\mathscr{F}$-decomposable elements of $A$ by $D(A, \mathscr{J})$ and we say that $\mathscr{J}$ is an unconditional decomposition for $A$ if $A=D(A, \mathscr{J})$.

If an element $x$ has an expansion as described above, then this expansion is necessarily unique, and also $\left\{\alpha \mid x_{\alpha} \neq 0\right\}$ is countable. If each $I_{\alpha}$ is one-dimensional, with generator $b_{\alpha}$, then $\mathscr{I}$ is an unconditional decomposition for $A$ if and only if $\left\{b_{\alpha}\right\}$ is an unconditional Schauder basis for $A[7, p .73]$.

Definition 3.2. For $x \in D(A, \mathscr{S})$, define

$$
|x|_{\mathscr{\sigma}}=\sup _{J \in \mathscr{G}}|| \sum_{\alpha \in J} x_{\alpha} \|
$$

where $\mathscr{F}$ denotes the collection of finite subsets of $C$.

It is straightforward to verify that $|\cdot| s$ defines a norm on $D(A, \mathscr{J})$, that $|x|_{\mathcal{S}} \geqq\|x\|, x \in D(A, \mathscr{F})$, and that $D(A, \mathscr{F})$ is complete in this new norm. When there is no confusion, we write $D$ for $D(A, \mathscr{J}),|\cdot|$ for $|\cdot|_{\mathcal{I}}$, and $\sum_{J}$ for $\sum_{\alpha \in J}$. We first prove the following:

\section{Proposition 3.3. $D(D(A, \mathscr{J}), \mathscr{J})=D(A, \mathscr{J})$.}

Proof. One sees that $\operatorname{cl}\left(I_{\alpha}\right)=I_{\alpha}$ and that $I_{\alpha} \cap \operatorname{cl}\left(\sum_{\beta \neq \alpha} I_{\beta}\right)=(0), \alpha \in C$, where the closure in both cases is taken with respect to the norm | |. Hence $D(D(A, \mathscr{I}), \mathscr{F})$ is well defined. If $x \in D, \varepsilon>0$, choose $J_{0} \varepsilon \mathscr{F}$ such that $J \cap J_{0}=\varnothing$ implies $\left\|\sum_{J} x_{\alpha}\right\|<\varepsilon$. If $J_{1} \in \mathscr{F}, J_{1} \supset J_{0}$, then $y=x-\sum_{J_{1}} x_{\alpha} \in D$ and it follows that $|y|=\sup _{J \in \mathscr{T}}\left\|\sum_{J-J_{1}} x_{\alpha}\right\| \leqq \varepsilon$. Thus $x=\sum x_{a}(|\cdot|)$, so $x \in D(D(A, \mathscr{F}), \mathscr{F})$.

We now give necessary and sufficient conditions on $\mathscr{F}$ which are equivalent to $\mathscr{F}$ being an unconditional decomposition for $A$.

THEOREM 3.4. Let $A$ be a Banach space, and let $\mathscr{J}=\left\{I_{\alpha} \mid \alpha \in C\right\}$ be a collection of closed subspaces with dense linear span such that $I_{\alpha} \cap \operatorname{cl}\left(\sum_{\beta \neq \alpha} I_{\beta}\right)=(0), \alpha \in C$. Then the following statements are equivalent: 
(1) $\mathscr{F}$ is an unconditional decomposition for $A$.

(2) The norms $\mid \cdot I_{\text {s }}$ and $\|\cdot\|$ are equivalent.

(3) There exists a constant $K$ such that, if $\alpha_{1}, \cdots, \alpha_{n}$ are distinct elements of $C$ and $x_{i} \in I_{\alpha_{i}}$, then

$$
\left\|\sum_{i=1}^{m} x_{i}\right\| \leqq K\left\|\sum_{i=1}^{n} x_{i}\right\|, 1 \leqq m \leqq n .
$$

(4) If $C=C_{1} \cup C_{2}$, with $C_{1} \cap C_{2}=\varnothing$, then there exists a constant $K\left(C_{1}, C_{2}\right)$ such that $x \in \sum_{C_{1}} I_{\alpha}, y \in \sum_{C_{2}} I_{\alpha}$ imply $\|x\| \leqq K\left(C_{1}, C_{2}\right)\|x+y\|$.

Proof. ${ }^{1}$ Since $D$ is dense in $A$, (1) is equivalent to $D$ being closed in $A$. Therefore the equivalence of (1) and (2) is given by [15, Corollary I.11] and the fact that $\|\cdot\| \leqq|\cdot|$. The equivalence of (1) and (3) follows from [15, Th. I.13].

Clearly (3) implies (4). Suppose that (4) does not imply (3). Then there exist disjoint finite subsets $\left\{B_{1}, C_{1}\right\} \subset C$, and elements $x_{1} \in \sum_{B_{1}} I_{\alpha}$, $y_{1} \in \sum_{C_{1}} I_{\alpha}$ such that $\left\|x_{1}\right\|>\left\|x_{1}+y_{1}\right\|$. Assume that pairwise disjoint finite subsets $\left\{B_{1}, \cdots, B_{n}, C_{1}, \cdots, C_{n}\right\} \subset C$ and elements $x_{1}, \cdots, x_{n}$, $y_{1}, \cdots, y_{n}$ have been chosen so that $x_{k} \in \sum_{B_{k}} I_{\alpha}, y_{k} \in \sum_{c_{k}} I_{\alpha}, 1 \leqq k \leqq n$ and such that $\left\|x_{k}\right\|>k\left\|x_{k}+y_{k}\right\|, 1 \leqq k \leqq n$. Let $E=\bigcup_{k=1}^{n}\left(B_{k} \cup C_{k}\right)$. Since $E$ is finite, $\sup _{G \subset E} K(G, C \sim G)<\infty$. Thus since (3) fails, there must exist disjoint subsets $\left\{B_{n+1}, C_{n+1}\right\} \subset C \sim E$ and elements $x_{n+1} \in \sum_{B_{n+1}} I_{\alpha}$, $y_{n+1} \in \sum_{C_{n+1}} I_{\alpha}$ such that $\left\|x_{n+1}\right\|>(n+1)\left\|x_{n+1}+y_{n+1}\right\|$. Now $x_{n+1}$ and $y_{n+1}$ are each contained in the linear span of a finite subset of $\left\{I_{\alpha} \mid \alpha \in C\right\}$, so $B_{n+1}$ and $C_{n+1}$ may be taken to be finite. Now let $H_{1}=\bigcup_{k=1}^{\infty} B_{k}$, $H_{2}=C \sim H_{1}$. Then $x_{n} \in H_{1}, y_{n} \in H_{2}$ for any $n$, so $K\left(H_{1}, H_{2}\right)>n$ for all $n$, which is a contradiction. Therefore (4) implies (3) and the theorem is established.

We now apply the preceding theorem to the case when $A$ is a semi-simple annihilator Banach algebra and $\mathscr{S}=\mathfrak{M}$.

THEOREM 3.5. Let $A$ be a semi-simple annihilator Banach algebra. Then the following statements are equivalent.

(1) The minimal-closed two-sided ideals of $A$ form an unconditional decomposition for $A$.

(2) $I \oplus \Re(I)=A$ for all closed two-sided ideals $I$.

Proof. We note that it is always true that $\operatorname{cl}(I \oplus \Re(I))=A$ for any closed two-sided ideal $I$.

(1) implies (2): Let $I$ be a closed two-sided ideal in $A$ and let $x \in A$. If $M \in \mathfrak{M}$, then $M \subset I$ or $M \subset \mathfrak{R}(I)$, and hence every summand in the infinite series expansion of $x$ is either in $I$ or $\mathfrak{R}(I)$. Thus $x \in I \oplus \Re(I)$.

1 The author is indebted to Professor R. C. James for suggestions which greatly shortened the original proof. 
(2) implies (1): It suffices to show that (4) of the preceding theorem holds, with $\mathscr{J}=\mathfrak{M}=\left\{M_{\alpha} \mid \alpha \in C\right\}$. Suppose $C=C_{1} \cup C_{2}$, with $C_{1} \cap C_{2}=\varnothing$, and let $J_{k}=\sum_{c_{k}} M_{\alpha}, k=1,2$. Then $\overline{J_{1}} \oplus \Re\left(\bar{J}_{1}\right)=A$, so it follows from the Closed Graph theorem that there exists a constant $K$ such that $\|u\| \leqq K\|u+v\|, u \in \bar{J}_{1}, v \in \Re\left(\bar{J}_{1}\right)$. Now $J_{2} \subset \mathfrak{R}\left(\bar{J}_{1}\right)$, so (4) is established.

Corollary 3.6. If A satisfies the conditions of the above theorem, and $I$ is a closed two-sided ideal in $A$, then

(1) $\mathfrak{R}(I)=I$.

(2) I is a semi-simple annihilator Banach algebra, with $\{M \in \mathfrak{M} \mid M \subset I\}$ its set of minimal-closed two-sided ideals, and $I$ satisfies the conditions of the above theorem.

Proof. If $x \in I$, then all of the summands in its infinite series expansion are in minimal-closed two-sided ideals which are contained in $I$. Hence $I=\operatorname{cl}\left(\sum_{M \subset I} M\right)$. The proof of the rest of the assertions is straightforward.

REMARK. The above theorem holds if $A$ is a proper $H^{*}$-algebra, a dual $B^{*}$-algebra, or $l_{p}, 1 \leqq p<\infty$.

We next apply Theorem 3.4 to the case when $A$ is a semi-simple annihilator Banach algebra with involution $x \rightarrow x^{*}$ such that $x x^{*}=0$ implies $x=0$ (such an involution is called proper), and $\mathscr{J}$ is a family of right ideals generated by a family of orthogonal hermitian idempotents. We note that if $\mathscr{I}=\left\{e_{\alpha} A \mid \alpha \in C\right\}$, where $\left\{e_{\alpha} \mid \alpha \in C\right\}$ is an orthogonal family of idempotents, and if $x$ is $\mathscr{I}$-decomposable, then the infinite series expansion of $x$ is given by $x=\sum_{\alpha} e_{\alpha} x$.

The proof of the following lemma is routine and is omitted.

LEMMA 3.7. Let $A$ be a semi-simple annihilator Banach algebra with proper involution. Then

(1) $\operatorname{cl}\left(I \oplus \mathbb{S}(I)^{*}\right)=A$ for all closed right ideals $I$.

(2) $\operatorname{cl}\left(I \oplus \Re(I)^{*}\right)=A$ for all closed left ideals $I$.

THEOREM. 3.8. Let $A$ be a semi-simple annihilator Banach algebra with proper involution. Then the following statements are equivalent.

(1) If $\left\{e_{\alpha}\right\}$ is a maximal family of orthogonal hermitian idempotents, then $x \in A$ implies $x=\sum_{\alpha} e_{\alpha} x$.

(2) If $\left\{e_{\alpha}\right\}$ is as in (1), then $x \in A$ implies $x=\sum_{\alpha} x e_{\alpha}$.

(3) $I \oplus \mathfrak{L}(I)^{*}=A$ for all closed right ideals $I$.

(4) $I \oplus \Re(I)^{*}=A$ for all closed left ideals $I$.

Moreover, the above conditions imply that $A$ is dual. 
Proof. (1) implies (3): Let $I$ be a closed right ideal in $A$, and let $\left\{e_{\alpha} \mid \alpha \in C_{1}\right\}$ be a maximal family of hermitian idempotents in $I$. Extend this family to a maximal such family in $A,\left\{e_{\alpha} \mid \alpha \in C\right\}$. Let $J=\mathrm{cl}\left(\sum_{\beta \notin C_{1}} e_{\beta} A\right)$. If $J \cap I \neq(0)$, then $J \cap I$ contains a hermitian idempotent $p$. But then $e_{\alpha} p=0, \alpha \in C_{1}$, which contradicts the maximality of $C_{1}$. Hence $x \in I$ implies $x-\sum_{C_{1}} e_{\alpha} x=\sum_{\alpha \oplus C_{1}} e_{\alpha} x \in I \cap J=(0)$, so $e_{\beta} x=0, \beta \notin C_{1}$. It follows that $\operatorname{cl}\left(\sum_{\beta \notin C_{1}} e_{\beta} A\right) \subset \mathcal{L}(I)^{*}$. Therefore $x \in A$ implies $x=\sum_{C_{1}} e_{\alpha} x+\sum_{\alpha \notin C_{1}} e_{\alpha} x \in I \oplus \mathfrak{L}(I)^{*}$.

(3) implies (1): If $\left\{e_{\alpha} \mid \alpha \in C\right\}$ is a maximal family of orthogonal hermitian idempotents, and $\mathscr{J}=\left\{e_{\alpha} A \mid \alpha \in C\right\}$, then the hypotheses of Theorem 3.4 are satisfied. The proof of (1) now follows in a manner analogous to the proof of "(2) implies (1)" in Theorem 3.5.

The other equivalences now follow from left-right symmetry and properties of the involution.

Suppose that one of conditions (1) through (4) holds, and let $I$ be a closed right ideal in $A$. Then $\mathscr{L}(I)^{*} \cap \mathfrak{L}\left(\mathcal{L}(I)^{*}\right)^{*}=(0)$. But $\mathscr{L}\left(\mathbb{L}(I)^{*}\right)^{*}=$ $\Re \mathbb{R}(I)$, and thus it follows that $I=\Re((I)$. Similarly if $I$ is a closed left ideal, then $I=\mathfrak{R}(I)$. Thus $A$ is dual.

REMARK. The above theorem holds if $A$ is a proper $H^{*}$-algebra or a dual $B^{*}$-algebra.

We conclude this section with an analysis of $D(A, \mathscr{J})$ in case $A$ is a Banach algebra and each $I_{\alpha}$ is a closed right ideal.

Lemma. 3.9. (1) $D$ is a dense right ideal in $A$ and $|x y| \leqq$ $|x| \cdot\|y\|, x \in D, y \in A$.

(2) If each $I_{\alpha}$ is two-sided, then $D$ is two-sided and $|x y| \leqq$ $\|x\| \cdot|y|, x \in A, y \in D$.

(3) $(D,|\cdot|)$ is a Banach algebra which is semi-simple if $A$ is semi-simple.

Proof. Since $D \supset \sum I_{\alpha}, D$ is dense in $A$. If $x \in D, y \in A$, then $x y=$ $\left(\sum_{\alpha} x_{\alpha}\right) y=\sum_{\alpha}\left(x_{\alpha} y\right)$. Therefore $x y \in D$. Since $\left\|\sum_{J} x_{\alpha} y\right\| \leqq|x| \cdot\|y\|$, $J \in \mathscr{F}$, we have that $|x y| \leqq|x| \cdot\|y\|$. Statement (2) is established in a similar manner.

From (1) it follows that $(D,|\cdot|)$ is a Banach algebra. Let $R$ be the radical of $D$. Then [8, p. 10] $R=\mathfrak{L}(D) \cap D \subset \mathfrak{L}(D)=\mathfrak{L}(\bar{D})=\mathfrak{L}(A)$. Hence $D$ is semi-simple if $A$ is.

Using the preceding lemma and the fact that a semi-simple Banach algebra is an annihilator algebra if it can be expressed as the topological sum of annihilator algebras, we have the following theorem. 
THEOREM. 3.10. Let $A$ be a semi-simple annihilator Banach algebra, and let $\mathscr{I}=\left\{I_{\alpha} \mid \alpha \in C\right\}$ be a collection of closed two-sided ideals of $A$. Suppose that either $\mathscr{J}=\mathfrak{M}$ or that $A$ is dual and equals the topological direct sum of $\mathscr{F}$. Then $\left(D(A, \mathscr{F}),|\cdot|_{\mathcal{I}}\right)$ is a semi-simple annihilator Banach algebra.

We now give an explicit characterization of $D(A, \mathfrak{M})$ in case $A=C(G)$ for $G$ a compact abelian topological group.

THEOREM. 3.11. Let $G$ be a compact abelian topological group, and let $A=C(G)$, with convolution for multiplication. Then $D(A, \mathfrak{M})$ is bi-continuously *-isomorphic to $L_{1}(\hat{G})$, where the latter has pointwise multiplication.

Proof. Let $\hat{G}=\left\{e_{\alpha}\right\}$, and let $M_{\alpha}=\left\{c e_{\alpha} \mid c\right.$ complex $\}$. Then $\mathfrak{M}=$ $\left\{M_{\alpha}\right\}$. Let $x \in D$. By considering real and imaginary parts, one shows that

$$
\sum_{\alpha}\left|x * e_{\alpha}(g)\right| \leqq 4|x|_{\mathfrak{M}}
$$$$
g \in G,
$$

where $*$ denotes convolution multiplication. Now $\hat{G}$ is discrete, so it follows that the left hand side of the above equation is just the $L_{1}$ norm of $\hat{x}$, the Fourier transform of $x$. Thus $x \rightarrow \widehat{x}$ is a continuous mapping of $D$ into $L_{1}(\widehat{G})$. It is straightforward to verify that the mapping is bicontinuous and a $*$-isomorphism onto $L_{1}(\hat{G})$.

Corollary. 3.12. If $A$ is as above, then $I \oplus \Re(I)=A$ for all closed ideals $I$ if and only if $G$ is finite.

Proof. If the first statement holds, then $D(A, \mathfrak{M})=A$. Thus the Fourier transform of every element of $A$ is in $L_{1}(\widehat{G})$. Thus, by the Inversion Theorem, every element of $C(G)$ is the Fourier transform of an element in $L_{1}(\hat{G})$, so by [16, Th. 4.6.8], $G$ is finite.

4. Structure and representation theorems. Throughout this section we assume that $A$ is a semi-simple annihilator Banach algebra. We investigate the structure of ideals and idempotents in $A$. The first lemma shows the relationship between $\mathfrak{M}$ and $\Pi$.

Lemma. 4.1. $\quad M \rightarrow \mathfrak{L}(M)$ is a one-to-one mapping of $\mathfrak{M}$ onto $I$. If $A$ is dual, then $P \rightarrow \mathfrak{R}(P)$ is a one-to-one mapping of $I$ onto $\mathfrak{M}$.

Proof. Let $M \in \mathfrak{M}$. Then there exists an idempotent $e \in M$ such that $M=\operatorname{cl}(A e A)$. Let $P=\{x \mid x A \subset A(1-e)\}$. Then $P \in \Pi$. Now $\mathfrak{L}(M) \subset \mathfrak{L}(e A)=A(1-e)$, and $\mathfrak{L}(M)$ is a closed two-sided ideal containing $P$. Therefore $\mathscr{L}(M)=P$. 
If $P \in \Pi$, then $\Re(P)$ contains a minimal idempotent $e$. Thus $M=\operatorname{cl}(A e A) \in \mathfrak{M}$ and $\mathfrak{L}(M)=P$. Thus $M \rightarrow \mathfrak{L}(M)$ is onto. Since the minimal-closed two-sided ideals annihilate each other, it follows that the mapping is one-to-one.

Suppose now that $A$ is dual. Let $P \in \Pi$ and let $M \in \mathfrak{M}$ such that $\mathfrak{L}(M)=P$. Then $\mathfrak{R}(P)=\mathfrak{R} \mathscr{L}(M)=M$. Thus $P \rightarrow \mathfrak{R}(P)$ is the inverse mapping of $M \rightarrow \mathfrak{L}(M)$ and hence is necessarily one-to-one and onto.

We next establish a representation theorem which essentially reduces to the Gelfand representation theorem when $A$ is commutative.

Lemma. 4.2. If $P \in \Pi$, then $A / P$ with the quotient norm is a primitive, topologically simple, annihilator Banach algebra.

Proof. The proof is straightforward and is omitted.

Definition. 4.3. Let $\mathscr{P} A / P$ denote the direct product of $\{A / P \mid P \in \Pi\}$, and let $\left(\sum A / P\right)_{0}$ denote the set of $f \in \mathscr{P} A / P$ such that $\{P \mid\|f(P)\| \geqq \varepsilon\}$ is finite for all $\varepsilon>0$. For $f \in\left(\sum A / P\right)_{0}$, define $\|f\|=$ $\sup _{P \in \Pi}\|f(P)\|$. Now, for $x \in A$, define $\hat{x} \in \mathscr{P} A / P$ by $\widehat{x}(P)=x+P$, and define the carrier of $x, \operatorname{car}(x)=\{P \mid \hat{x}(P) \neq 0\}$.

THEOREM 4.4. $\left(\sum A / P\right)_{0}$ is a semi-simple annihilator Banach algebra, and $x \rightarrow \widehat{x}$ is a norm-decreasing isomorphism of $A$ onto $a$ dense subset of $\left(\sum A / P\right)_{0}$.

Proof. The proof of the first statement is sketched in [14, pp. 106-7]. It is easily seen that $x \rightarrow \hat{x}$ is an isomorphism of $A$ into $\mathscr{P} A / P$. Let $x \in A, \varepsilon>0$. Then there exist $M_{i} \in \mathfrak{M}$ and $x_{i} \in M_{i}$, $1 \leqq i \leqq n$, such that $\left\|x-\sum_{i=1}^{n} x_{i}\right\|<\varepsilon$. Let $P_{i}=\mathscr{L}\left(M_{i}\right)$; then car $\left(x_{i}\right) \subset\left\{P_{i}\right\}$. Thus $P \notin\left\{P_{1}, \cdots, P_{n}\right\}$ implies

$$
\|\widehat{x}(P)\|=\left\|\left(x-\sum_{i=1}^{n} x_{i}\right) \hat{(P)}\right\|<\varepsilon,
$$

so $\hat{x} \in\left(\sum A / P\right)_{0}$.

Since $\|\hat{x}(P)\| \leqq\|x\|$, the mapping is norm-decreasing. Thus it remains to show that the range of the mapping is dense in $\left(\sum A / P\right)_{0}$. It is sufficient to show that the set of $\hat{x}$ with finite carriers is dense in the direct sum of $\{A / P \mid P \in \Pi\}$, since this latter set is dense in $\left(\sum A / P\right)_{0}$. So, suppose $f \in\left(\sum A / P\right)_{0}$, that $f$ vanishes except for $P \in\left\{P_{1}, \cdots, P_{n}\right\}$, and let $\varepsilon>0$. Now choose $M_{i} \in \mathfrak{M}$ such that $\mathbb{L}\left(M_{i}\right)=$ $P_{i}$. Since $\operatorname{cl}\left(M_{i}+P_{i}\right)=A$, the image of $M_{i}$ is dense under the natural map from $A$ onto $A / P_{i}$. Thus there exist $x_{i} \in M_{i}$ such that $\left\|\widehat{x}_{i}\left(P_{i}\right)-f\left(P_{i}\right)\right\|<\varepsilon, 1 \leqq i \leqq n$. If $x=\sum_{i=1}^{n} x_{i}$, then $\operatorname{car}(x) \subset\left\{P_{1}, \cdots, P_{n}\right\}$ and $\|\widehat{x}-f\|=\sup _{1 \leqq i \leqq n}\left\|\widehat{x}_{i}\left(P_{i}\right)-f\left(P_{i}\right)\right\|<\varepsilon$. 
REMARK. In general, the mapping $x \rightarrow \hat{x}$ is not bicontinuous. However, if $A$ is a $B^{*}$-algebra, then $A$ is isometrically ${ }^{*}$-isomorphic to $\left(\sum A / P\right)_{0}[11$, Th. 8.1].

We now turn our attention to the decomposability of idempotents.

THEOREM. 4.5. Every idempotent in $A$ can be expressed as the sum of orthogonal minimal idempotents.

Proof. Let $e$ be an idempotent in $A$. First, suppose that $e$ is contained in some $M \in \mathfrak{M}$. Let $I$ be a minimal left ideal in $M$ and let $x \rightarrow L(x)$ be the left regular representation of $M$ as compact operators on $I$ (see [4, Th. 9 and 10]). If $S=$ range $(L(e)$ ), then $S$ is closed and $L(e)$ is the identity on $S$. Hence $S$ is finite-dimensional. Thus there exist orthogonal bounded projections with one-dimensional range, $E_{1}, \cdots, E_{n}$ such that $E_{1}+\cdots+E_{n}=L(e)$. Since the range of $x \rightarrow L(x)$ includes all bounded operators on $I$ with finite-dimensional range, each $E_{k}$ is the image of some $e_{k} \in M$. Thus $\left\{e_{1}, \cdots, e_{n}\right\}$ is a set of orthogonal minimal idempotents, and $e=e_{1}+\cdots+e_{n}$.

Now, suppose that $e$ is any idempotent in $A$. Then $\hat{e}$ is an idempotent in $\left(\sum A / P\right)_{0}$ and hence $\operatorname{car}(e)$ is finite, say $\operatorname{car}(e)=$ $\left\{P_{1}, \cdots, P_{n}\right\}$. Let $M_{k} \in \mathfrak{M}, \mathfrak{R}\left(M_{k}\right)=P_{k}$. It is straightforward to verify that every idempotent in $A / P_{k}$ is the image of some idempotent $q_{k} \in M_{k}$ under the natural map from $A$ onto $A / P_{k}$. Hence $\widehat{q}_{k}\left(P_{k}\right)=\hat{e}\left(P_{k}\right)$, and by the first part each $q_{k}$ is the sum of orthogonal minimal idempotents. Since $M_{k} M_{j}=(0), k \neq j$, it follows that $e=q_{1}+\cdots+q_{n}$ and hence that $e$ is the sum of orthogonal minimal idempotents.

The following corollary has been shown by Barnes [3] for the more general class of modular annihilator algebras.

Corollary. 4.6. Let $e$ be an idempotent in $A$. Then eAe is finite-dimensional. Hence $A$ is finite-dimensional if it has an identity.

Proof. If $e$ and $f$ are minimal idempotents, then e $A f$ is (0) or one-dimensional.

Using the above corollary, we now establish the following result about $\Xi$, the set of maximal-modular two-sided ideals.

Proposition. 4.7. The following statements are equivalent.

(1) $A$ is strongly semi-simple.

(2) $\Pi=\Xi$.

(3) Each $M \in \mathfrak{M}$ is finite-dimensional.

(4) Each $M \in \mathfrak{M}$ has identity. 
Proof. If $P_{0} \in \Pi \sim \Xi$, and $M \in \mathfrak{M}, \mathbb{R}(M)=P_{0}$, then $M \subset \cap\{P \mid P \in \Xi\}$. Therefore (1) implies (2). If (2) holds and $M \in \mathfrak{M}$, then $\mathfrak{L}(M) \in \Xi$, so $A / \mathscr{L}(M)$ has identity and hence is finite-dimensional. The mapping $x \rightarrow \widehat{x}(\mathscr{L}(M))$ is one-to-one on $M$, so $M$ is finite-dimensional. Therefore (2) implies (3). If (3) holds and $M \in \mathfrak{M}$, then (see Theorem 4.5) $M$ is isomorphic to all the operators on any of its minimal left ideals. Hence $M$ has identity. Thus (3) implies (4). If $M \in \mathfrak{M}$, then $\mathfrak{L}(M)$ is maximal-closed. If (4) holds, then $\mathscr{L}(M)$ is also modular, so $\Pi=$ $\{\mathfrak{Q}(M) \mid M \in \mathfrak{M}\}=\Xi$. Therefore (4) implies (2). Clearly (2) implies (1).

The rest of this section consists of structure theorems for certain kinds of one-sided ideals in $A$. Although the theorems are stated and proved only for right ideals, the corresponding statements for left ideals also hold.

TheOREM. 4.8. Let $e_{1}, \cdots, e_{n}$ be idempotents in $A$. Then there exists an idempotent $e \in A$ such that $e A=e_{1} A+\cdots+e_{n} A$.

Proof. We note that if $e_{1}, \cdots, e_{n}$ are orthogonal, then $e$ can be taken as $e_{1}+\cdots+e_{n}$.

It suffices to prove the theorem for $n=2$, since the general case then follows by induction. First, suppose that $e_{1}$ and $e_{2}$ are both contained in the same $M \in \mathfrak{M}$. Let $x \rightarrow L(x)$ be as in the proof of Theorem 4.5 and let $S_{k}=$ range $\left(L\left(e_{k}\right)\right)$. Then $S_{1}, S_{2}$ are finitedimensional, so there exist bounded projections $P_{1}, P_{2}$ such that range $\left(P_{1}\right)=S_{1}$ and range $\left(P_{2}\right)=S_{2} \sim S_{1}$. Thus $E=P_{1}+P_{2}$ is a bounded projection, so there exists an idempotent $e \in M$ such that $L(e)=E$. Now $L(e) L\left(e_{k}\right)=L\left(e_{k}\right)$, so $e_{1} A+e_{2} A \subset e A$. Also, $L\left(e_{k}\right) P_{k}=$ $P_{k}$, so it follows that $e A \subset e_{1} A+e_{2} A$.

Now suppose that $e_{1}$ and $e_{2}$ are any idempotents in $A$. Since any minimal idempotent is contained in some $M \in \mathfrak{M}$, it follows from Theorem 4.5 that $e_{1}=p_{1,1}+\cdots+p_{n, 1}$ and $e_{2}=p_{1,2}+\cdots+p_{n, 2}$, where each $p_{k, j}$ is an idempotent in $M_{k} \in \mathfrak{M}, j=1,2,1 \leqq k \leqq n$. By applying the previous case to each pair $\left\{p_{k, 1}, p_{k, 2}\right\}$ and using the fact that $M_{i} M_{j}=(0), i \neq j$, the theorem is established.

As a consequence of Theorems 4.5 and 4.8 , we obtain the following:

Corollary. 4.9. The socle of $A$ is the union of the idempotentgenerated right ideals of $A$.

We conclude this section with an analysis of a special class of right ideals which arise in the study of homomorphisms. If $y \in A$ we denote by $J(y)$ the closed right ideal $\{x \mid y x=x\}$. The following fact about $J(y)$ will be used in establishing Theorem 5.1. 
Lemma. 4.10. Let $y \in A$ and let $G=\{P\|\| \hat{y}(P) \| \geqq 1\}$. Then $G$ is a finite set, and car $(x) \subset G$ for all $x \in J(y)$.

Proof. If $0 \neq x \in J(y), P \in \operatorname{car}(x)$, then $\hat{y}(P) \hat{x}(P)=\hat{x}(P) \neq 0$. Therefore $\|\hat{y}(P)\| \geqq 1$, so $P \in G$. Since $\hat{y} \in\left(\sum A / P\right)_{0}, G$ is finite.

THEOREM. 4.11. $J(y)$ is idempotent-generated for all $y \in A$.

Proof. First, suppose that $J(y)$ is contained in some $M \in \mathfrak{M}$, and let $x \rightarrow L(x)$ be as in the proof of Theorem 4.5. If $S=\{x \mid L(y) x=x\}$, then $S$ is closed and $L(y)$ is the identity on $S$, so $S$ is finite-dimensional. Thus there exists a bounded projection $E$ with range $S$, and so there exists an idempotent $e \in M$ such that $L(e)=E$. It follows that $y e=e$, and hence $e A \subset J(y)$. If $u \in J(y)$, then $L(y) L(u)=L(u)$, so $L(e) L(u)=$ $L(u)$. Therefore $u \in e A$.

If $y$ is any element of $A$, let $\{P \mid\|\hat{y}(P)\| \geqq 1\}=\left\{P_{1}, \cdots, P_{n}\right\}$. Since $A / P_{k}$ is topologically simple, by the previous case there exist idempotents $q_{k} \in A / P_{k}$ such that $q_{k}\left(A / P_{k}\right)=J\left(\hat{y}\left(P_{k}\right)\right)$. Thus there exists an idempotent $e \in A$ with carrier $\left\{P_{1}, \cdots, P_{n}\right\}$ such that $\hat{e}\left(P_{k}\right)=q_{k}$, $1 \leqq k \leqq n$. It follows that $e A=J(y)$.

Corollary 4.12. The socle of $A$ is the set of elements possessing a left identity.

5. Continuity properties of homomorphisms. We now apply the results of the previous two sections to the study of continuity properties of homomorphisms of semi-simple annihilator Banach algebras. Our first objective is to establish the following analog for annihilator algebras of a theorem by Bade and Curtis [2, Th. 3.7].

THEOREM 5.1. Let $A$ be a semi-simple annihilator Banach algebra in which $I \oplus \Re(I)=A$ for all closed two-sided ideals $I$, and let $\nu$ be a homomorphism of $A$ into a Banach algebra. Then there exists a finite set of primitive ideals, $\Pi_{0}$, and a constant $K$ such that

$$
\|\nu(x)\| \leqq K\|x\| \cdot\|y\|
$$

for all $x$ and $y$ in $A$ such that $y x=x$ and $\operatorname{car}(x) \subset \Pi \sim \Pi_{0}$.

The proof of this theorem will be given after several lemmas have been established. If $A$ is any semi-simple annihilator Banach algebra, then it will be possible to make a slightly weaker conclusion, but with $\|\cdot\|$ replaced by $|\cdot|_{\mathfrak{M}}$.

We will make repeated use of the following theorem, which was proved in the commutative case by Bade and Curtis [2]. 
Theorem 5.2. Let $A$ and $B$ be Banach algebras, and let $\nu$ be a homomorphism of $A$ into $B$. If $\left\{x_{n}\right\}$ and $\left\{y_{n}\right\}$ are sequences in $A$ such that

$$
y_{n} x_{n}=x_{n}
$$

and

$$
y_{n} x_{m}=0, \quad n \neq m,
$$

then

$$
\sup _{n}\left\|\nu\left(x_{n}\right)\right\| /\left\|x_{n}\right\| \cdot\left\|y_{n}\right\|<\infty
$$

Proof. This is proved in [6, Corollary 3.2]. The hypotheses in [2] and [6] have (2) replaced by the stronger condition $y_{n} y_{m}=0$, $n \neq m$. However, this is not necessary.

In proving Theorem 5.1, we will use a construction which is basically the same as that in [2]. Through Lemma 5.8, we will assume that $A$ is a semi-simple annihilator Banach algebra in which $I \oplus \Re(I)=A$ for all closed two-sided ideals $I$, and that $\nu$ is a homomorphism of $A$ into a Banach algebra. By Theorems 3.4 and 3.5 , the norms $\|\cdot\|$ and $|\cdot|_{\mathfrak{M}}$ are equivalent. Since we are dealing with continuity properties of $\nu$, there is no loss of generality in assuming that these norms are numerically equal. We will do so as it simplifies the computations.

Definition 5.3. Let $\mathscr{K}$ be the set of $E \subset \Pi$ such that $\sup \{\|\nu(x)\| /\|x\| \cdot\|y\|: y x=x, \operatorname{car}(x) \cup \operatorname{car}(y) \subset E\} \equiv K(E)<\infty$.

We will show that $\mathscr{C}$ has a largest element whose complement is finite.

Lemma 5.4. If $\left\{E_{j}\right\}$ is a pairwise disjoint family of subsets of $I$, then there exists a constant $K$ such that $K\left(E_{\delta}\right) \leqq K$, and hence $E_{o} \in \mathscr{Y}$, for all but finitely many $\delta$.

Proof. Suppose the conclusion is false. Then there exists a distinct sequence $\left\{E_{\delta_{n}}\right\}$ and sequences $\left\{x_{n}\right\}$ and $\left\{y_{n}\right\}$ such that $y_{n} x_{n}=x_{n}$, $\operatorname{car}\left(x_{n}\right) \cup \operatorname{car}\left(y_{n}\right) \subset E_{\delta_{n}}$ and $\left\|\nu\left(x_{n}\right)\right\| /\left\|x_{n}\right\| \cdot\left\|y_{n}\right\|>n$. But $\operatorname{car}\left(y_{n} x_{m}\right) \subset$ $E_{\hat{o}_{n}} \cap E_{\hat{o}_{m}}=\phi, n \neq m$, so that $y_{n} x_{m}=0, n \neq m$, which contradicts Theorem 5.2.

Lemma 5.5. Suppose that $E_{1}$ and $E_{2}$ are disjoint subsets of $\Pi$ and that $x$ and $y$ are elements of $A$ such that $y x=x$ and car $(x) \cup$ $\operatorname{car}(y) \subset E_{1} \cup E_{2}$. Then for $k=1,2$ there exist $x_{k}, y_{k} \in A$ such that 


$$
\begin{gathered}
y_{k} x_{k}=x_{k}, \quad \operatorname{car}\left(x_{k}\right) \cup \operatorname{car}\left(y_{k}\right) \subset E_{k} \\
x_{1}+x_{2}=x, \quad y_{1}+y_{2}=y \\
\left\|x_{k}\right\| \leqq\|x\|, \quad\left\|y_{k}\right\| \leqq\|y\| .
\end{gathered}
$$

Proof. Since $x$ is $\mathfrak{M}$-decomposable, and car $(x) \subset E_{1} \cup E_{2}, x$ has an infinite series expansion in which each summand has its one element carrier either in $E_{1}$ or $E_{2}$. Hence $x$ can be factored into $x_{1}+x_{2}$ where car $\left(x_{k}\right) \subset E_{k}$, and similarly for $y$. Since $y x=x$ and $E_{1} \cap E_{2}=\phi$, it follows that $y_{k} x_{k}=x_{k}$. Statement (3) follows from the fact that $\|\cdot\|=|\cdot|$.

Lemma 5.6. If $\left\{E_{1}, \cdots, E_{n}\right\} \subset \mathscr{C}$, then $\bigcup_{k=1}^{n} E_{k} \in \mathscr{C} \quad$ and $K\left(\bigcup_{k=1}^{n} E_{k}\right) \leqq \sum_{k=1}^{n} K\left(E_{k}\right)$.

Proof. It suffices to establish the conclusion for $n=2$, since the general case then follows by induction. Without loss of generality, we may assume $E_{1} \cap E_{2}=\dot{\phi}$.

Suppose that $y x=x$ and $\operatorname{car}(x) \cup \operatorname{car}(y) \subset E_{1} \cup E_{2}$. Now chose $x_{k}$ and $y_{k}$ as in the previous lemma. Then

$$
\begin{aligned}
\frac{\|\nu(x)\|}{\|x\| \cdot\|y\|} & \leqq \frac{\left\|\nu\left(x_{1}\right)+\nu\left(x_{2}\right)\right\|}{\|x\| \cdot\|y\|} \leqq \frac{\left\|\nu\left(x_{1}\right)\right\|}{\left\|x_{1}\right\| \cdot\left\|y_{1}\right\|}+\frac{\left\|\nu\left(x_{2}\right)\right\|}{\left\|x_{2}\right\| \cdot\left\|y_{2}\right\|} \\
& \leqq K\left(E_{1}\right)+K\left(E_{2}\right),
\end{aligned}
$$

and hence the conclusion follows.

Lemma 5.7. There exists a constant $K$ such that $K(E) \leqq K$ for all $E \in \mathscr{C}$.

Proof. Suppose the conclusion is false. Then there exists a sequence $\left\{E_{k}\right\} \subset \mathscr{H}$ such that $K\left(E_{1}\right)>1$ and $K\left(E_{k}\right)>(k+1) K\left(E_{k-1}\right)$. Let $A_{1}=E_{1}$ and let $A_{n}=E_{n} \sim \bigcup_{k=1}^{n-1} E_{k}$. Then $\left\{A_{n}\right\}$ is a pairwise disjoint sequence in $\mathscr{C}$. Now $\bigcup_{k=1}^{n-1} E_{k} \in H$ and $\left(\bigcup_{k=1}^{n-1} E_{k}\right) \cup A_{n}=E_{n}$. Thus, using the preceding lemma, it follows that $K\left(A_{n}\right)>2 K\left(E_{n-1}\right) \geqq 2 K\left(A_{n-1}\right)$, so $K\left(A_{n}\right)>2^{n-1}$, which contradicts Lemma 5.4 .

Lemma 5.8. $\cup \mathscr{C}$ is an element of $\mathscr{H}$.

Proof. Let $E_{0}$ be the union of everything in $\mathscr{H}$. Suppose that $y x=x$ and that $\operatorname{car}(x) \cup \operatorname{car}(y) \subset E_{0}$. Then by Lemma 4.10, car $(x)$ is finite, so it is contained in the union of a finite number of elements of $\mathscr{C}$, say $E_{1}$. Now $E_{1} \in \mathscr{C}$, and in view of Lemma 5.5 there is no loss of generality in assuming that $\operatorname{car}(y) \subset E_{1}$. Thus if $K$ is as in 
Lemma 5.7, then $\|\nu(x)\| /\|x\| \cdot\|y\| \leqq K\left(E_{1}\right) \leqq K$. Thus $K\left(E_{0}\right) \leqq K$ and $E_{0} \in \mathscr{Y}$.

Proof of Theorem 5.1. Let $E_{0}$ and $K$ be as in previous lemmas and let $\Pi_{0}$ be the complement of $E_{0}$. By Lemma 5.4, $\Pi_{0}$ has only finitely many elements. Suppose that $y x=x$ and $\operatorname{car}(x) \subset E_{0}$. Now choose $y_{1}$ such that $y_{1} x=x$, car $\left(y_{1}\right) \subset E_{0}$ and $\left\|y_{1}\right\| \leqq\|y\|$. Then $\|\nu(x)\| \leqq K\|x\| \cdot\left\|y_{1}\right\| \leqq K\|x \mid \cdot\| y \|$.

CoROllaRY 5.9. If $A$ is strongly semi-simple, then

$$
\|\nu(x)\| \leqq K\|x\| \cdot\|y\|
$$

for all $x$ and $y$ such that $y x=x$.

Proof. Let $P \in \Pi=\Xi$, and choose $M \in \mathfrak{M}$ such that $\mathfrak{L}(M)=P$. Then $M$ is finite-dimensional, so $\nu \mid M$ is continuous. Therefore $\{P\} \in \mathscr{C}$. Thus $\Pi \in \mathscr{H}$.

EXAMPLE. If $\nu$ is the discontinuous isomorphism of $l_{2}$ constructed in [2], and if $p_{n}$ is the sequence whose first $n$ terms are one and the rest zero, then $p_{n}=p_{n}^{2}$ and $\left\|\nu\left(p_{n}\right)\right\|=n=\left\|p_{n}\right\|^{2}$.

The next lemma shows that is $A$ if any semi-simple annihilator Banach algebra, and $\nu$ is a homomorphism of $A$ into a Banach algebra, then we can apply Theorem 5.1 to $\nu$ by considering it as a homomorphism of $D(A, \mathfrak{M})$.

LEMma 5.10. Let $A$ be a semi-simple annihilator Banach algebra. Let $\Pi_{D}$ denote the primitive ideals and $\mathfrak{M}_{D}$ the minimal-closed two-sided ideals of $D(A, \mathfrak{M})$. Then

$$
\begin{gathered}
\mathfrak{M}_{D}=\mathfrak{M}, \\
\Pi_{D}=\{P \cap D(A, \mathfrak{M}) \mid P \in \Pi\},
\end{gathered}
$$

and

(3) $\quad\left(D(A, \mathfrak{M}),|\cdot|_{\mathfrak{M}}\right)$ is a semi-simple annihilator Banach algebra in which $I \oplus R(I)=D(A, \mathfrak{M})$ for all closed two-sided ideals $I$.

Proof. The first assertion follows from the fact that $\|\cdot\|=|\cdot|$ on each $M_{\alpha} \in \mathfrak{M}$ and that $\operatorname{cl}\left(\sum_{\alpha} M_{\alpha}\right)=A$. By Theorem $3.10,(D,|\cdot|)$ is a semi-simple annihilator Banach algebra. Hence (2) follows from (1) and Lemma 4.1. Statement (3) follows from (1), Proposition 3.3, and Theorem 3.5. 
THEOREM 5.11. Let $A$ be a semi-simple annihilator Banach algebra, and let $\nu$ be a homomorphism of $A$ into a Banach algebra. Then (1) If $y x=x$, then $x \in D(A, \mathfrak{M})$ and there exists $y_{1} \in D(A, \mathfrak{M})$ such that $y_{1} x=x$.

(2) There exists a constant $K$ and a finite set of primitive ideals $I_{0}$ such that

$$
\|\nu(x)\| \leqq K|x|_{\mathfrak{M}} \cdot|y|_{\mathfrak{M}}
$$

for all $x$ and $y$ in $A$ such that $y x=x, \operatorname{car}(x) \subset \Pi \sim \Pi_{0}$ and $y \in D(A, \mathfrak{M})$.

Proof. The first assertion follows from Theorem 4.11 and its corollary. By the preceding lemma, we can apply Theorem 5.1 to $\nu$ considered as a homomorphism of $D$. Statement (2) now follows from this and the fact that primitive ideals in $D$ are just the intersection with $D$ of primitive ideals in $A$.

6. The separating ideal. If $A$ and $B$ are Banach algebras, and $\nu$ is a homomorphism of $A$ onto a dense subset of $B$, than define the separating ideal of $\nu$ in $B$,

$$
S=\left\{\mathrm{y} \in B \mid \inf _{x \in A}\{\|x\|+\|\nu(x)-y\|\}=0\right\} .
$$

The separating ideal was first introduced by Rickart [13]. For basic properties of $S$, see [18] or [6]. If $A$ is a semi-simple annihilator Banach algebra, then we have the following.

Proposition 6.1. Let $S_{1}=\nu^{-1}(S)$. Then

(1) $S_{1} \cdot A \subset \operatorname{cl}\left(\nu^{-1}(0)\right)$.

(2) If $A$ is dual, then $S_{1}=\operatorname{cl}\left(\nu^{-1}(0)\right)$.

(3) If $\nu$ is an isomorphism, then $S_{1}=(0)$.

Proof. Let $K=\nu^{-1}(0)$. Let $M \in \mathfrak{M}$. If $M \cap S_{1}=(0)$ then $S_{1} M \subset$ $M \cap S_{1} \subset K$. Otherwise, $M \cap S_{1}=M$, so $M \subset S_{1}$ : If $e$ is an idempotent in $M$ then $e \in S_{1}$, so by a theorem of Yood [6, Th. 4.8] $\nu(e)=0$. Thus the socle of $M$ is contained in $K$, so $M \subset \bar{K}$. Thus $S_{1} M \subset \bar{K}$ for all $M \in \mathfrak{M}$ so (1) follows.

If $A$ is dual, then $S_{1}$ is a semi-simple dual Banach algebra. Now the socle of $S_{1}$ is contained in $K$ and hence $S_{1} \subset \bar{K}$. Clearly $\bar{K} \subset S_{1}$, so (2) holds.

If $\nu$ is an isomorphism, then by (1), $S_{1} \subset \mathfrak{L}(A)=(0)$.

7. Homomorphisms of dual $\mathbf{B}^{*}$-algebras. In this section we specialize to the case when $A$ is a dual $B^{*}$-algebra. By using a theorem of Johnson [9] which says that any homomorphism of the 
compact operators on Hilbert space into a Banach algebra is continuous, we can strengthen Theorem 5.1 in this case. Throughout this section, $F$ denotes the socle of $A$.

TheOREM 7.1. Let $A$ be a dual $B^{*}$-algebra, and let $\nu$ be a homomorphism of $A$ into a Banach algebra. Then $\nu$ is continuous on $F$.

Proof. By Theorem 5.1 there exists a constant $K$ and a finite set of primitive ideals $\Pi_{0}$ such that $\|\nu(x)\| \leqq K\|x\| \cdot\|y\|$ whenever $y x=x$ and $\operatorname{car}(x) \subset \Pi \sim \Pi_{0}$.

Let $x \in F$. Then by Corollary 4.9 there exists an idempotent $e$ such that $e x=x$. By Theorem 4.5 there exist orthogonal minimal idempotents $e_{1}, \cdots, e_{n}$ such that $e=e_{1}+\cdots+e_{n}$. Now any minimal left ideal in $A$ is a Hilbert space in the given norm and $x \rightarrow L(x)$ is a *-isomorphism. Thus an examination of the proof of Theorem 4.5 in this special case shows that the one-dimensional subspaces, range $\left(L\left(e_{k}\right)\right)$, can be taken as mutually orthogonal, and hence the $e_{k}$ can be taken as hermitian. Thus we may (and do) assume that $e$ is a hermitian idempotent, and hence that $\|e\|=1$.

Now, let $\Pi_{0}=\left\{P_{1}, \cdots, P_{m}\right\}$, and choose $M_{k} \in \mathfrak{M}$ such that $\mathfrak{L}\left(M_{k}\right)=P_{k}$. Then $x=x_{0}+\cdots+x_{m}$, where $x_{k} \in M_{k}, 1 \leqq k \leqq m$, and $x_{0}$ is in the sum of the remaining members of $\mathfrak{M}$. Thus $e x_{0}=x_{0}$ and $\operatorname{car}\left(x_{0}\right) \subset \Pi \sim \Pi_{0}$, so $\left\|\nu\left(x_{0}\right)\right\| \leqq K\left\|x_{0}\right\|$. Each $M_{k}$ is isometrically *-isomorphic to the compact operators on a Hilbert space, so by the theorem of Johnson, $\nu$ is continuous on each $M_{k}$. Since $\left\|x_{k}\right\| \leqq\|x\|, 0 \leqq k \leqq m$, it follows that there exists a constant $K^{\prime}$ independent of $x$ such that $\|\nu(x)\| \leqq K^{\prime}\|x\|$.

REMARK. Since $\nu \mid F$ is continuous and $\bar{F}=A$, there exists a unique continuous homomorphism of $A$ onto a (not necessarily dense) subset of $\operatorname{cl}(\nu(A))$ which agrees with $\nu$ on $F$.

We now establish two decomposition theorems about the range of an isomorphism of a dual $\mathrm{B}^{*}$-algebra. These theorems are analogous to a theorem of Bade and Curtis on homomorphisms of commutative $\mathrm{B}^{*}$-algebras [2, Th. 4.3].

Theorem 7.2. Let $A$ be a dual $B^{*}$-algebra, and let $\nu$ be an isomorphism of $A$ onto a dense subset of a Banach algebra $B$. Let $\mu$ be the continuous homomorphism of $A$ into $B$ which agrees with $\nu$ on $F$, let $\lambda=\nu-\mu$, and let $S$ be the separating ideal for $\nu$ in $B$. Then

(1) $\mu$ is an isomorphism

(2) $S=\operatorname{cl}(\lambda(A))$ 
(3) $\mu(A)$ is a closed two-sided ideal in $B$ and $B=\mu(A) \oplus S$

(4) $\mu(A) S=S \mu(A)=(0)$ and $\lambda$ is a homomorphism.

Proof. (1) By [6, Lemma 5.3] there exists a constant $L$ such that $\|x\| \leqq L\|\nu(x)\|, x \in A$. Suppose $\mu(x)=0$. Then there exists a sequence $\left\{x_{n}\right\} \subset F$ such that $x_{n} \rightarrow x$. Hence $\nu\left(x_{n}\right)=\mu\left(x_{n}\right) \rightarrow \mu(x)=0$, so $x_{n} \rightarrow 0$. Therefore $\mu$ is an isomorphism.

(2) Let $x \in A$ and let $\left\{x_{n}\right\} \subset F, x_{n} \rightarrow x$. Then $\nu\left(x_{n}\right)=\mu\left(x_{n}\right) \rightarrow \mu(x)$. Thus $x-x_{n} \rightarrow 0, \nu\left(x-x_{n}\right) \rightarrow \lambda(x)$, so $\lambda(A) \subset S$. If $y \in S$ and $x_{n} \rightarrow 0$, $\nu\left(x_{n}\right) \rightarrow y$, then $\lambda\left(x_{n}\right)=(\nu-\mu)\left(x_{n}\right) \rightarrow y-0 \in S$, so $S \subset \operatorname{cl}(\lambda(A))$.

(3) By [6, p. 1104], $\mu(A)$ is closed and $B=\nu(A) \oplus S$. If $x \in A$, $y \in F$, then $\nu(x) \mu(y)=\nu(x y)=\mu(x y) \in \mu(A)$. Therefore $\nu(A) \mu(A) \subset$ $\operatorname{cl}(\mu(A))=\mu(A)$, and similarly $\mu(A) \nu(A) \subset \mu(A)$, so $\mu(A)$ is a two-sided ideal in $B$. If $\mu(x) \in S$, then $\nu(x)=(\lambda+\mu)(x) \in S$, so $\nu(x)=0=x$. Therefore $\mu(A) \cap S=(0)$. Thus $B=\nu(A) \oplus S=(\lambda+\mu)(A) \oplus S \subset \mu(A) \oplus S$, so $B=\mu(A) \oplus S$.

(4) The first statement follows directly from (3). If $x, y \in A$ then $\lambda(x) \mu(y)=\mu(x) \lambda(y)=0$, so

$$
\begin{aligned}
\lambda(x) \lambda(y) & =((\mu+\lambda)(x)((\mu+\lambda)(y))-\mu(x y) \\
& =(\nu-\mu)(x y)=\lambda(x y) .
\end{aligned}
$$

Therefore $\lambda$ is a homomorphism.

Before proving the second decomposition theorem, we digress to establish the following theorem about homomorphisms of a large class of $\mathrm{B}^{*}$-algebras. This was proved in the commutative case by Yood [17, Corollary 5.5].

THEOREM 7.3. Let $A$ be a $B^{*}$-algebra in which every primitive ideal is modular. Let $\nu$ be a homomorphism of $A$ into a Banach algebra $B$ such that $\mathrm{cl}(\nu(A))=B$ and $\nu^{-1}(0)$ is closed. Let $R$ denote the strong radical of $B$.

Then $R=S$ and $B=\nu(A) \oplus R$.

Proof. We note that the hypotheses for $A$ will be satisfied if $A$ has identity, if $A$ is commutative, or if $A$ is a strongly semi-simple dual algebra.

First, suppose that $\nu$ is one-to-one. By a theorem of Cleveland [6, Th. 5.5] $B=\nu(A) \oplus S$, and by a theorem of Yood [18, Th. 3.5] $R \supset S$. Hence to complete the proof for this case we only need show that $\nu(A) \cap R=(0)$.

Now there exists a constant $L$ such that $\|x\| \leqq L\|\nu(x)\|, x \in A$. Let $P \in \Xi$ and let $e$ be a two-sided identity for $A$ modulo $P$. If $\operatorname{cl}(\nu(P))=B$ then there exists a sequence $\left\{x_{n}\right\} \subset P$ such that $\nu\left(x_{n}\right) \rightarrow \nu(e)$. 
Thus $x_{n} \rightarrow e$, so $e \in P$, which is a contradiction. Thus $\mathrm{cl}(\nu(P))$ is a proper modular two-sided ideal and hence is contained in a maximal such ideal, $J_{P}$. It follows that $\nu^{-1}\left(J_{P}\right)=P$ and hence that

$$
(0)=\cap\left\{\nu^{-1}\left(J_{P}\right) \mid P \in \Xi\right\} \supset \nu^{-1}(R) \text {. }
$$

Hence the conclusion follows if $\nu$ is one-to-one.

If $\nu^{-1}(0)$ is closed, then $A_{1}=A / \nu^{-1}(0)$ is a $B^{*}$-algebra. Let $\pi$ be the natural map from $A$ onto $A_{1}$. By considering inverse images under $\pi$, we see that every primitive ideal of $A_{1}$ is modular. Also, it is straightforward to show that the mapping $\nu_{1}$ defined by $\nu_{1} \circ \pi=\nu$ is an isomorphism of $A_{1}$ onto $\nu(A)$. By the first case,

$$
B=\nu_{1}\left(A_{1}\right) \oplus R=\nu(A) \oplus R .
$$

Let $S_{1}$ be the separating ideal for $\nu_{1}$ in $B$. Since $\pi$ is continuous, $S \subset S_{1}$. On the other hand, suppose there exists a sequence $\left\{x_{n}\right\} \subset A$ such that $\pi\left(x_{n}\right) \rightarrow 0$ and $\nu_{1}\left(\pi\left(x_{n}\right)\right) \rightarrow y \in B$. Then there exists a sequence $\left\{w_{n}\right\} \subset \nu^{-1}(0)$ such that $\left\|x_{n}+w_{n}\right\| \rightarrow 0$. But $\nu\left(x_{n}+w_{n}\right)=\nu\left(x_{n}\right) \rightarrow y$, so $y \in S$. Thus $S=S_{1}=R$, and the theorem is established.

As a direct consequence of the preceding two theorems, we have the following.

THEOREM 7.4. Let $A$ be a strongly semi-simple dual $B^{*}$-algebra, and let $\nu$ be an isomorphism of $A$ onto a dense subset of a Banach algebra $B$. Let $\mu$ be the continuous isomorphism of $A$ into $B$ which agrees with $\nu$ on $F$, let $\lambda=\nu-\mu$, and let $R$ denote the strong radical of $B$. Then

(1) $\operatorname{cl}(\lambda(A))=R$.

(2) $\mu(A)$ is a closed two-sided ideal in $B$ and $B=\mu(A) \oplus R$.

(3) $R \mu(A)=\mu(A) R=(0)$, and $\lambda$ is a homomorphism.

\section{REFERENCES}

1. W. Ambrose, Structure theorems for a special class of Banach algebras, Trans. Amer. Math. Soc. 57 (1945), 364-386.

2. W. G. Bade and P.C. Curtis, Jr., Homomorphisms of commutative Banach algebras, Amer. J. Math. 82 (1960), 589-608.

3. B. A. Barnes, Modular annihilator algebras, Canad. J. Math. 18 (1966), 566-578.

4. F. F. Bonsall and A.W. Goldie, Annihilator algebras, Proc. London Math. Soc. (3) 4 (1954), 154-167.

5. P. Civin and B. Yood, Lie and Jordan structures in Banach algebras, Pacific J. Math. 15 (1965), 775-797.

6. S. B. Cleveland, Homomorphisms of non-commutative *-algebras, Pacific J. Math. 13 (1963), 1097-1109.

7. M. M. Day, Normed Linear Spaces, Academic Press Inc., New York, 1962. 
8. N. Jacobson, Structure of Rings, Amer. Math. Soc. Colloq. Publ. no. 37, Providence, 1956.

9. B. E. Johnson, Continuity of homomorphisms of algebras of operators, J. London Math. Soc. 42 (1967), 537-541.

10. I. Kaplansky, Dual rings, Ann. of Math. (2) 49 (1948), 689-701.

11. - Normed algebras, Duke Math. J. 16 (1949), 399-418.

12. M. A. Naimark, Normed Rings, P. Noordhoff, Groningen, 1959.

13. C. E. Rickart, The uniqueness of norm problem in Banach algebras, Ann. of Math.

(2) 51 (1950), 615-628.

14. - General Theory of Banach Algebras, Van Nostrand, New York, 1960.

15. W. H. Ruckle, The infinite sum of closed subspaces of an F-space, Duke Math. J.

31 (1964), 543-554.

16. W. Rudin, Fourier Analysis on Groups, Interscience Publishers, New York, 1962.

17. B. Yood, Topological properties of homomorphisms between Banach algebras, Amer. J. Math. 76 (1954), 155-167.

18. - Homomorphisms on normed algebras, Pacific J. Math. 8 (1958), 373-381.

Received November 10, 1966. This paper is taken from the author's doctoral dissertation, which was written under the direction of Professor Paul Civin. This research was supported in part by National Science Foundation grant GP-3927.

UNIVERSITY OF OREGON AND

State University of NeW York at Stony Brook 



\section{PACIFIC JOURNAL OF MATHEMATICS}

\section{EDITORS}

\section{H. ROYDEN}

Stanford University

Stanford, California
J. DugundJI

Department of Mathematics

Rice University

Houston, Texas 77001

RICHARD ARENS

University of California

Los Angeles, California 90024

Seattle, Washington 98105

\section{ASSOCIATE EDITORS}
E. F. BeCKENBACH
B. H. NeumanN
F. WOLF
K. YOSIDA

\section{SUPPORTING INSTITUTIONS}

\author{
UNIVERSITY OF BRITISH COLUMBIA \\ CALIFORNIA INSTITUTE OF TECHNOLOGY \\ UNIVERSITY OF CALIFORNIA \\ MONTANA STATE UNIVERSITY \\ UNIVERSITY OF NEVADA \\ NEW MEXICO STATE UNIVERSITY \\ OREGON STATE UNIVERSITY \\ UNIVERSITY OF OREGON \\ OSAKA UNIVERSITY \\ UNIVERSITY OF SOUTHERN CALIFORNIA
}

\author{
STANFORD UNIVERSITY \\ UNIVERSITY OF TOKYO \\ UNIVERSITY OF UTAH \\ WASHINGTON STATE UNIVERSITY \\ UNIVERSITY OF WASHINGTON \\ * * * * \\ AMERICAN MATHEMATICAL SOCIETY \\ CHEVRON RESEARCH CORPORATION \\ TRW SYSTEMS \\ NAVAL WEAPONS CENTER
}

Mathematical papers intended for publication in the Pacific Journal of Mathematics should be in typed form or offset-reproduced, double spaced with large margins. Underline Greek letters in red, German in green, and script in blue. The first paragraph or two must be capable of being used separately as a synopsis of the entire paper. It should not contain references to the bibliography. Manuscripts, in duplicate if possible, may be sent to any one of the four editors. All other communications to the editors should be addressed to the managing editor, Richard Arens, University of California, Los Angeles, California 90024.

Each author of each article receives 50 reprints free of charge; additional copies may be obtained at cost in multiples of 50 .

The Pacific Journal of Mathematics is published monthly. Effective with Volume 16 the price per volume (3 numbers) is $\$ 8.00$; single issues, $\$ 3.00$. Special price for current issues to individual faculty members of supporting institutions and to individual members of the American Mathematical Society: $\$ 4.00$ per volume; single issues $\$ 1.50$. Back numbers are available.

Subscriptions, orders for back numbers, and changes of address should be sent to Pacific Journal of Mathematics, 103 Highland Boulevard, Berkeley 8, California.

Printed at Kokusai Bunken Insatsusha (International Academic Printing Co., Ltd.), 7-17, Fujimi 2-chome, Chiyoda-ku, Tokyo, Japan.

PUBLISHED BY PACIFIC JOURNAL OF MATHEMATICS, A NON-PROFIT CORPORATION

The Supporting Institutions listed above contribute to the cost of publication of this Journal, but they are not owners of publishers and have no responsibility for its content or policies. 


\section{Pacific Journal of Mathematics \\ Vol. 25, No. $2 \quad$ October, 1968}

Martin Aigner, On the tetrahedral graph ..................... 219

Gregory Frank Bachelis, Homomorphisms of annihilator Banach

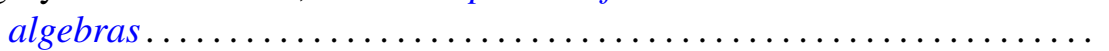

Phillip Alan Griffith, Transitive and fully transitive primary abelian groups.......................................... 249

Benjamin Rigler Halpern, Fixed points for iterates . . . . . . . . . . . . . 255

James Edgar Keesling, Mappings and dimension in general metric spaces ......................................... 277

$\mathrm{Al}$ (Allen Frederick) Kelley, Jr., Invariance for linear systems of ordinary differential equations ................................ 289

Hayri Korezlioglu, Reproducing kernels in separable Hilbert spaces . . . . . 305

Gerson Louis Levin and Wolmer Vasconcelos, Homological dimensions and Macaulay rings ................................. 315

Leo Sario and Mitsuru Nakai, Point norms in the construction of harmonic

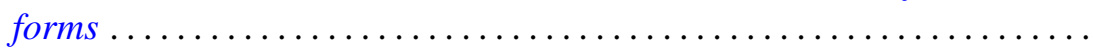

Barbara Osofsky, Noncommutative rings whose cyclic modules have cyclic injective hulls ..................................... 331

Newton Tenney Peck, Extreme points and dimension theory........... 341

Jack Segal, Quasi dimension type. II. Types in 1-dimensional spaces ...... 353

Michael Schilder, Expected values of functionals with respect to the Ito distribution ...

Grigorios Tsagas, A Riemannian space with strictly positive sectional curvature

John Alexander Williamson, Random walks and Riesz kernels . . 